\title{
Dosis de refuerzo con la vacuna BNT162b2 en población que recibió el esquema de vacunación completa para COVID-19 en Perú: Un análisis crítico de la evidencia actual ${ }^{\star}$
}

\section{Booster dose with the BNT162b2 vaccine in the population that received the complete immunization schedule for COVID-19 in Peru: A critical analysis of the current evidence}

DOI

https://doi.org/10.35434/rcmhnaaa.2021.14Sup1.1177

\section{RESUMEN}

Introducción: En el Perú, el programa actual de inmunización para COVID-19 comprende las vacunas BBIBP-CorV, BNT162B2 y ChAdOx1 nCoV-19. Si bien el esquema de inmunización es de dos dosis, algunos países han incluido recientemente una dosis de refuerzo a su esquema. Métodos: Se realizó una búsqueda de evidencia científica sobre la eficacia y seguridad de la vacunación de refuerzo con la vacuna BNT162b2 en población con esquema de vacunación completa para COVID-19 en Perú. Evidencia incluida: Se consideraron cuatro documentos de recomendación basados en evidencia, un estudio observacional y tres ensayos clínicos fase III en curso. Conclusión: A la fecha, no existe evidencia suficiente sobre la eficacia de agregar una dosis de refuerzo al esquema de inmunización para COVID-19. La evidencia disponible no permite justificar el uso de una dosis de refuerzo con la vacuna BNT162B2 en población que recibió previamente dos dosis de las vacunas anteriormente mencionadas.

Palabras Clave: Infecciones por Coronavirus; Vacunación; Inmunización Secundaria(Fuente: DeCS-BIREME).

\section{ABSTRACT}

Background: In Peru, the current immunization schedule for COVID-19 includes BBIBP-CorV, BNT162B2 and ChAdOx1 nCoV-19 vaccines. Although the full immunization schedule is two doses, some countries have recently included a booster dose to their schedule. Methods: We conducted a search for scientific evidence on the efficacy and safety of booster vaccination with BNT162b2 vaccine in a population with a complete vaccination schedule for COVID-19 in Peru. Evidence: Four evidence-based recommendation documents, one observational study and three ongoing phase III clinical trials were included for analysis. Conclusion: To date, there is insufficient evidence on the efficacy of adding a booster dose to the immunization schedule for COVID-19. The available evidence does not justify the use of a booster dose of BNT162B2 vaccine in a population that has previously received two doses of the aforementioned vaccines.

Keywords: Coronavirus Infections; COVID-19 Vaccines; Immunization, Secondary. (Source: MeSH-NLM).
Carlos J. Toro-Huamanchumo ${ }^{1,2, a}$, Jose A. Zavala-Loayza ${ }^{1, a}$, Raisa N. Martinez-Rivera ${ }^{3,4, b}$, Verónica Peralta ${ }^{1, c}$, Eric R. Peña-Sanchez ${ }^{1,5, a}$

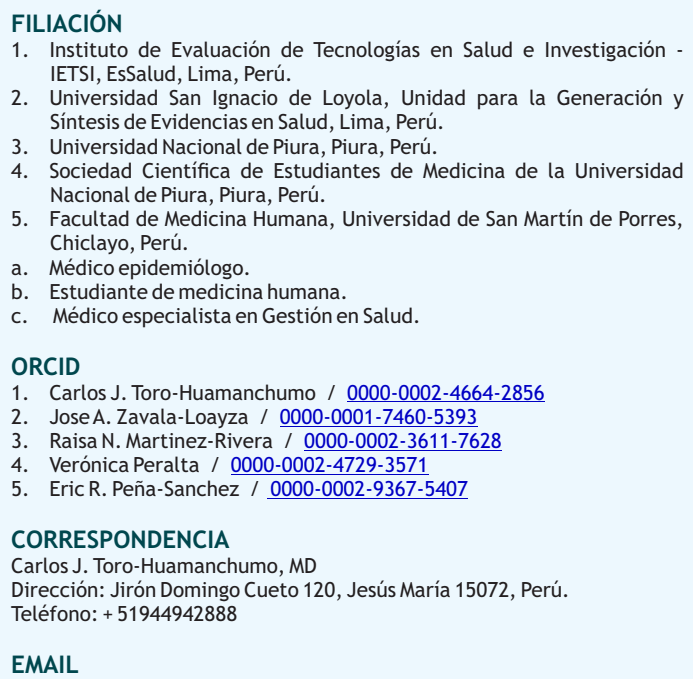

CJTH, JAZL, VP y ERPS son funcionarios del IETSI. Asimismo, CJTH y ERPS son miembros del comité editor de la Revista Del Cuerpo Médico Hospital Nacional Almanzor Aguinaga Asenjo, y no tuvieron participación en ninguna etapa del proceso editorial posterior al envío del presente artículo. RNMR declara no tener conflictos de interés.

\section{FINANCIAMIENTO}

Seguro Social de Salud (EsSalud)

\section{CONTRIBUCIÓN DEAUTORÍA}

CJTH, JAZL, VP y ERPS concibieron la idea de investigación. CJTH y RNMR redactaron el primer borrador del manuscrito. Todos los autores revisaron críticamente el manuscrito, dieron aportes sustanciales, aprobaron la versión final y asumen responsabilidad relacionada a todos los aspectos del artículo

\section{REVISIÓN DE PARES}

Recibido: 30/08/2021

Aceptado: 07/09/2021

\section{COMO CITAR}

Toro-Huamanchumo, C., Zavala-Loayza, J., Martinez-Rivera, R., Peralta, V., \& Peña-Sánchez, E. Dosis de refuerzo con la vacuna BNT162b2 en población que recibió el esquema de vacunación completa para COVID-19 en Perú: Un análisis crítico de la evidencia actual*. Revista Del Cuerpo Médico Hospital Nacional Almanzor Aguinaga Asenjo, 2021, 14(Sup1), 70 78. https://doi.org/10.35434/rcmhnaaa.2021.14Sup1.1177

\section{(i)}

Esta obra está bajo una Licencia Creative Commons Atribución 4.0 Internacional. Versión Impresa: ISSN: 2225-5109 Versión Electrónica: ISSN: 2227-4731

Cross Ref. DOI: $10.35434 /$ rcmhnaaa

OJS: https://cmhnaaa.org.pe/ojs 


\section{INTRODUCCIÓN}

Al 13 de septiembre de 2021, se han administrado más de 5,534 millones de dosis de vacuna contra COVID-19 a nivel mundial ${ }^{(1)}$. En el Perú, desde que inició la vacunación (segunda semana de febrero del 2021) hasta la fecha (setiembre de 2021), hay más de 21 millones de dosis aplicadas y casi 9 millones de personas con vacunación completa $^{(2)}$.

Actualmente, el esquema de inmunización completa para COVID-19 está compuesto por dos dosis de las vacunas elaboradas por los laboratorios Pfizer-BioNTech ${ }^{(3)}$, Moderna ${ }^{(4)}$, Sinovac ${ }^{(5)}$, Sinopharm ${ }^{(6)}$, Novavax ${ }^{(7)}$ y Oxford-Astra Zeneca ${ }^{(8)}$. En el caso de Johnson \& Johnson/Janssen, el esquema comprende una sola dosis ${ }^{(9)}$. Sin embargo, algunos países de Europa $^{(10)}$, Asia ${ }^{(11)}$ y América ${ }^{(12)}$ han comenzado a incluir una dosis adicional a su esquema de inmunización. En América Latina, países como República dominicana ${ }^{(13)}$, Chile ${ }^{(14)}$ y Uruguay $^{(15)}$ actualmente tienen aprobada una tercera dosis con las vacunas de Pfizer y AstraZeneca, para su aplicación en personas vacunadas previamente con Sinovac.

Los términos “tercera dosis”, “dosis de refuerzo” ("booster”) y "dosis adicional" no son intercambiables. El primero hace únicamente referencia al número de dosis que la persona recibe; pudiendo ser de refuerzo o adicional. Los Centros para el Control y la Prevención de Enfermedades (CDC, por sus siglas en inglés) definen a la dosis de refuerzo como aquella que es administrada cuando es probable que, pese a que inicialmente se consiguió una respuesta inmunitaria suficiente con el esquema completo de vacunación, esta haya disminuido con el tiempo ${ }^{(16)}$. Por su parte, la dosis adicional es definida como aquella que es administrada cuando es probable que la respuesta inmunitaria, después del esquema completo, sea insuficiente; por ejemplo, en personas con grado de inmunosupresión moderado a grave ${ }^{(17)}$.

Recientemente, el Instituto de Evaluación de Tecnologías en Salud e Investigación (IETSI) realizó una búsqueda de evidencia científica sobre la eficacia y seguridad de la vacunación de refuerzo con la vacuna BNT162b2 (Pfizer), en personas con inmunización completa con la vacuna BBIBP. CorV (Sinopharm) $)^{(18)}$. El informe concluyó que, al 28 de junio de 2021, no se disponía de evidencia que respalde esta dosis de refuerzo. Sin embargo, en el Perú, además de la BBIBPCorV, el programa de inmunización para COVID-19 incluye las vacunas BNT162b2 y ChAdOx1 nCoV-19 (AstraZeneca), desde marzo y abril, respectivamente ${ }^{(19)}$. Además, considerando la posibilidad de que nueva evidencia se haya publicado desde la realización del reporte del IETSI hasta la fecha, se decidió realizar una revisión sistemática de la literatura con el objetivo de evaluar la eficacia y seguridad de la dosis de refuerzo con la vacuna BNT162b2, en población que haya recibido el esquema de inmunización completa con la vacuna BBIBP-CorV, BNT162b2 o ChAdOx1 nCoV-19.

\section{MÉTODOS}

\section{Pregunta Clínica (PICO)}

Para cumplir con el objetivo de la presente investigación, se formuló una pregunta PICO que incluya desenlaces de eficacia y seguridad (Tabla 1 ).

\section{Búsqueda de la evidencia}

Se realizó una búsqueda sistemática de la evidencia publicada hasta el 27 de agosto del 2021 en las bases de datos de PubMed, Cochrane Library y LILACS (Literatura Latinoamericana y del Caribe en Ciencias de la Salud), así como en los repositorios de preimpresiones MedRxiv, BioRxiv,

Tabla 1. Pregunta PICO elaborada por el equipo evaluador del IETSI.

\begin{tabular}{|c|c|}
\hline Población & Personas con esquema de vacunación completa contra COVID-19 en Perú* \\
\hline Intervención & Dosis de refuerzo** con la vacuna BNT162b2† \\
\hline Comparador & Sin dosis de refuerzo \\
\hline \multirow{9}{*}{ Outcome (Desenlace) } & Eficacia: \\
\hline & -Mortalidad \\
\hline & •Hospitalizaciones \\
\hline & -Ingreso a unidad de cuidados intensivos (UCl) \\
\hline & •Necesidad de ventilación mecánica \\
\hline & •Casos confirmados $† \dagger$ sintomáticos de COVID-19 (cualquier severidad $\ddagger$ ) \\
\hline & •Casos confirmadosł† asintomáticos de COVID-19 \\
\hline & Seguridad: \\
\hline & •Eventos adversos \\
\hline
\end{tabular}

*Incluye la vacunación con dos dosis de BBIBP-CorV, BNT162b2 o ChAdOx1 nCoV-19.

*Dosis de vacuna administrada cuando es probable que la respuesta inmunitaria inicial suficiente, después de la vacunación primaria, haya disminuido con el tiempo (16).

†Vacuna de tipo ARN mensajero (ARNm) contra COVID-19, desarrollada por BioNTech en colaboración con Pfizer.

tCon resultado de reacción en cadena de la polimerasa con retrotranscripción (RT-PCR) positivo para SARS-CoV-2.

¥Según definición propuesta por la Organización Mundial de la Salud (OMS) (34). Casos leves: pacientes sintomáticos que cumplen la definición de caso de COVID-19 sin evidencia de neumonía viral o hipoxia. Casos moderados: pacientes con signos clínicos de neumonía (fiebre, tos, disnea, respiración rápida), pero sin signos de neumonía grave, incluyendo una saturación de oxígeno ( $\mathrm{SpO2}$ ) 290\% en aire ambiente. Casos severos: pacientes con signos clínicos de neumonía (fiebre, tos, disnea, respiración rápida) más uno de los siguientes: frecuencia respiratoria $>30$ respiraciones/min, dificultad respiratoria severa o $\mathrm{SpO2} \mathrm{<90 \%} \mathrm{en} \mathrm{aire} \mathrm{ambiente.} \mathrm{Casos} \mathrm{criticos:} \mathrm{pacientes} \mathrm{con} \mathrm{criterios} \mathrm{para} \mathrm{sindrome} \mathrm{de} \mathrm{dificultad} \mathrm{respiratoria}$ aguda (SDRA), sepsis, shock séptico u otras afecciones que normalmente requerirían la provisión de terapias de soporte vital, como ventilación mecánica (invasiva o no invasiva) o terapia vasopresora. 
Revista del Cuerpo Médico del HNAAA, Vol. 14 Supl. №1 ( 2021 ) | SARS-CoV-2, COVID-19 y Pandemia

Research Square y SSRN. Las estrategias de búsqueda junto con los resultados obtenidos pueden visualizarse en las tablas S1-S6 del material suplementario.

La búsqueda sistemática fue complementada con una búsqueda manual en la lista de referencias bibliográficas de los estudios incluidos en la evaluación a texto completo. Además, se realizó una búsqueda de literatura gris en el motor de búsqueda Google y se realizó una búsqueda dentro de las páginas web pertenecientes a grupos que realizan guías de práctica clínica (GPC) y evaluaciones de tecnologías sanitarias (ETS), incluyendo el National Institute for Health and Care Excellence (NICE), la Agency for Healthcare Research and Quality's (AHRQ), la Scottish Intercollegiate Guidelines Network (SIGN), la Guidelines International Network (GIN), el Scottish Medicines Consortium (SMC), la Canadian Agency for Drugs and Technologies in Health (CADTH), el Institut für Qualität und Wirtschaftlichkeit im Gesundheitswesen (IQWIG), la Haute Autorité de santé (HAS), la Agenzia nazionale per $i$ servizi sanitari regionali (AGENAS), el Centro Nacional de Excelencia Tecnológica en Salud (CENETEC), la Comissão Nacional de Incorporação de Tecnologias no Sistema Único de Saúde (CONITEC), el Instituto de Evaluación Tecnológica en Salud (IETS) y el Instituto de Efectividad Clínica y Sanitaria (IECS). También se revisó la base de datos de la International Network of Agencies for Health Technology Assessment (INAHTA) y la Base Regional de Informes de Evaluación de Tecnologías en Salud de las Américas (BRISA).

Otras fuentes de información incluyeron las páginas web de la Organización Mundial de la Salud (OMS), la Organización Panamericana de Salud, los CDC de Estados Unidos, el Centro Europeo para la Prevención y Control de Enfermedades (ECDC, por sus siglas en inglés), la FDA, la Agencia Europea de Medicamentos (EMA, por sus siglas en inglés), el Ministerio de Salud del Perú y la Dirección General de Medicamentos, Insumos y Drogas (DIGEMID). Por último, se realizó una búsqueda de estudios clínicos en curso o aún no publicados en ClinicalTrials.gov, el International Clinical Trial Registry Platform (ICTRP) y el Registro Nacional de Proyectos de Investigación en Salud (PRISA) del Instituto Nacional de Salud (INS) del Perú.

\section{Criterios de selección}

Se incluyeron documentos en español o inglés. La búsqueda se enfocó inicialmente en la identificación de GPC, ETS y ensayos clínicos aleatorizados (ECA) de fase III. En el caso de las GPC, se buscó seleccionar a aquellas cuyo proceso de desarrollo fue sistemático y estaba descrito, que realizaron una búsqueda y revisión de evidencia científica, que incluyeron algún sistema de gradación para la fuerza de recomendación y el nivel de evidencia, y que presentaran recomendaciones para la población específica de la pregunta PICO del presente estudio. De no encontrarse, se procedió con la selección de documentos de recomendación basados en la evidencia. Para su inclusión, debían al menos mencionar que hicieron una búsqueda y revisión de la evidencia científica y tener conclusiones o recomendaciones enfocadas en nuestra población de interés. En el caso de las ETS, se buscó incluir a aquellas que correspondían con la población e intervención de la pregunta PICO, cuyo proceso de desarrollo fue sistemático y estaba descrito, que realizaron una búsqueda y revisión de evidencia científica, y que hayan finalizado con una recomendación o toma de decisión; o al menos que contenían un análisis crítico de la evidencia incluida. Por último, respecto a los estudios primarios, de no encontrarse ECA fase III publicados, se planteó la inclusión de estudios observacionales, reportes o series de caso y reportes de fármacovigilancia que hayan evaluado la pregunta PICO de interés. También se incluyeron ECA fase III en curso y/o no publicados bajo la misma consideración.

\section{Proceso de selección}

La selección de los estudios fue llevada a cabo en tres fases (Figura 1). La primera fue realizada por dos evaluadores de manera independiente y consistió en la revisión de los títulos y resúmenes. Todas las discrepancias se resolvieron por consenso. La segunda fase fue realizada por un evaluador y consistió en la revisión a texto completo de los estudios preseleccionados, con la intención de incluir a aquellos que cumplieron con los criterios de elegibilidad. Esta fase también incluyó el tamizaje de los registros identificados en la literatura gris. Por último, la tercera fase consistió en la selección final de los documentos incluidos en el presente estudio.

\section{RESULTADOS}

Se incluyeron en el presente reporte un total de ocho documentos (Figura 1). Se identificaron cuatro documentos de recomendación basados en evidencia y un estudio observacional. Se incluyeron también tres ensayos clínicos fase III en curso o aún sin publicarse a la fecha del presente manuscrito (13 de septiembre de 2021). Se excluyeron siete estudios observacionales que no respondieron a la pregunta PICO planteada (Tabla S7 del material suplementario).

\section{Descripción y evaluación de la evidencia}

\section{i) Documentos de recomendación basados en la evidencia}

European Centre for Disease Prevention and Control. "Interim public health considerations for the provision of additional COVID-19 vaccine doses" (20)

Se trata de las recomendaciones provisionales de salud pública para el suministro de vacunación de refuerzo contra COVID-19, elaborado por el ECDC. El reporte técnico fue publicado el 01 de septiembre de 2021.

La evaluación de la evidencia sobre la efectividad de las vacunas para SARS-CoV-2 se basó en la revisión sistemática "viva" ("living systematic review") de la Universidad de John Hopkins y la $\mathrm{OMS}^{(21)}$, la síntesis de evidencia viva ("living evidence synthesis") de la Red de Evidencia COVID-END del Foro de Salud de la Universidad McMaster ${ }^{(22)}$, y un reporte técnico previo del ECDC sobre vacunación parcial y completa ${ }^{(23)}$.

De acuerdo con la evidencia revisada, el ECDC concluye que existe un buen nivel de efectividad de las diferentes vacunas autorizadas en la Unión Europea (que incluyen dos de las vacunas consideradas en la PICO del presente estudio: Pfizer y AstraZeneca). En otras palabras, ofrecen protección para enfermedad sintomática, enfermedad severa, 


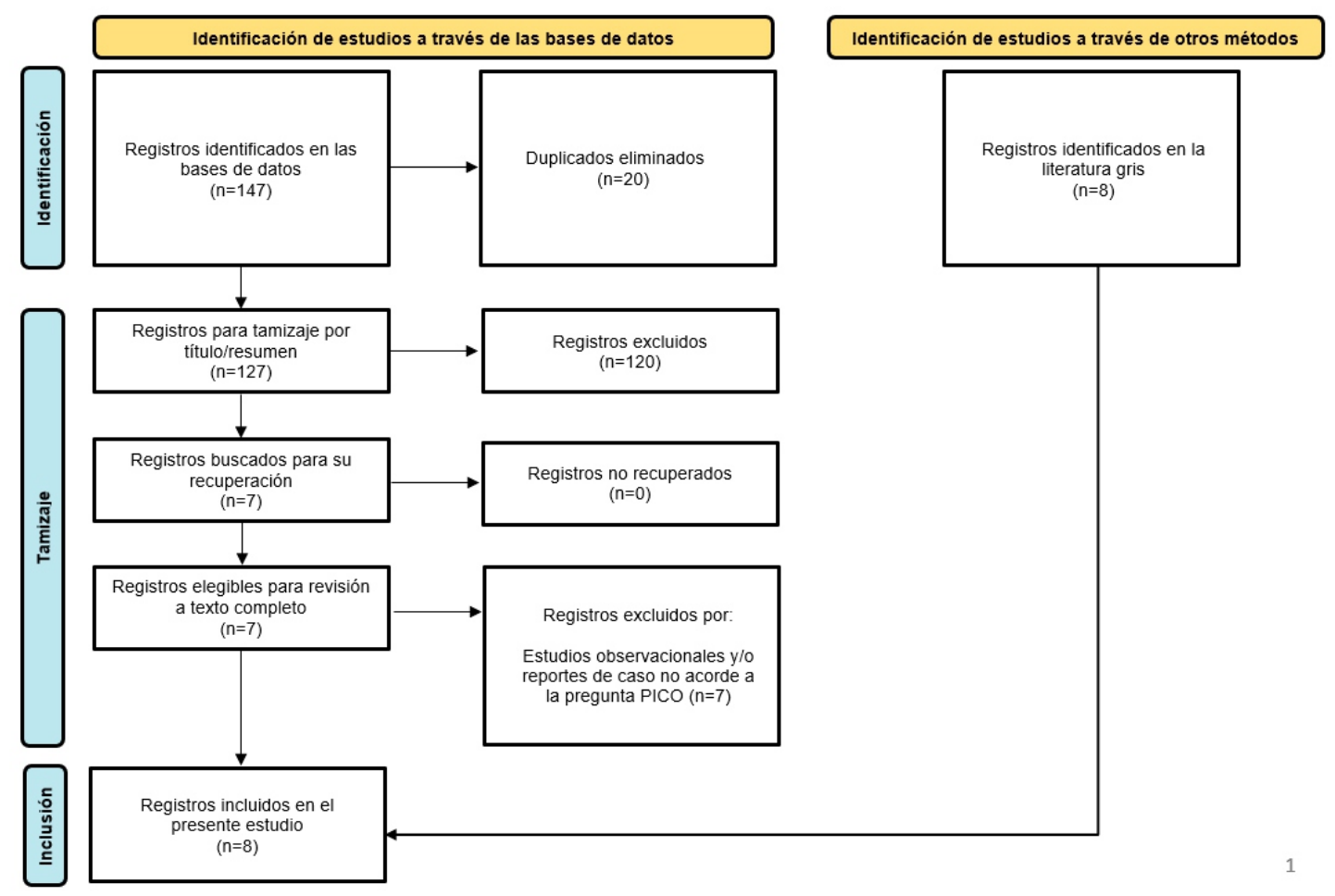

Figura 1.

Flujograma de selección de la bibliografía encontrada

hospitalización y muerte, después de un esquema de inmunización completo, en la población en general. EI ECDC mantuvo esta conclusión incluso para las variantes de preocupación (VOC, por sus siglas en inglés), que incluyen la B.1.1.7 (Alfa), B.1.351 (Beta), P.1 (Gamma) y B.1.617.2 (Delta).

El ECDC sostiene que la evaluación de una posible dosis de refuerzo para la COVID-19, desde la perspectiva de salud pública, debería tener como principal objetivo la prevención de casos severos. Sugieren también que este sea el desenlace primario de los estudios que evalúen la necesidad de dosis adicionales en grupos específicos.

Finalmente, según la evidencia disponible, el ECDC considera que no existe una necesidad urgente de la administración de dosis de refuerzo en la población en general. Sin embargo, sí refieren que la opción de administrar dosis adicionales en individuos que puedan experimentar una respuesta limitada a la vacunación primaria (i.e. pacientes inmunosuprimidos) debería ser considerada. En este sentido, esta opción debe verse como parte del esquema de vacunación para estos grupos específicos y no como un refuerzo para aquellos que sí desarrollaron una respuesta inmune adecuada. En el caso de adultos de edad avanzada que además cumplan los criterios de fragilidad y vivan en casas de reposo o asilos, podría considerarse la posibilidad de suministrar, como precaución, una dosis adicional.

World Health Organization. "Interim statement on COVID19 vaccine booster doses" (24)

Se trata de las recomendaciones provisionales de la OMS sobre el uso de dosis de refuerzo de las vacunas para COVID19, a través del Grupo de Expertos de Asesoramiento Estratégico sobre Inmunización (SAGE, por sus siglas en inglés) y su Grupo de Trabajo de Vacunas COVID-19. Las recomendaciones están actualizadas al 10 de agosto de 2021 y se extienden a todas las vacunas incluidas en el listado de uso en emergencias. El documento no detalla cuál fue la evidencia usada para la emisión de sus recomendaciones.

Las conclusiones del documento derivan de la evaluación de tres factores. Primero, la disminución de la inmunidad ("waning immunity"). La OMS sostiene que, hasta la fecha, no se ha establecido un correlato inmunitario de protección (ni de su duración), el cual se define como un marcador inmunitario específico que se asocie a la protección contra la infección ${ }^{(25)}$. Si bien algunos estudios sugieren una posible relación entre la eficacia de las vacunas contra la enfermedad sintomática y la inducción de títulos medios de anticuerpos neutralizantes, no está claro si la disminución de los títulos a lo largo del tiempo es indicativa de la disminución de la eficacia de la vacuna. Asimismo, si bien puede haber una pérdida de protección contra las infecciones por SARS-CoV-2, la OMS sostiene que es probable que la protección contra la enfermedad severa se mantenga en niveles considerables debido a la inmunidad celular.

Segundo, la efectividad de las vacunas. La OMS sostiene que la evidencia actualmente disponible es insuficiente para definir si existe una disminución significativa de la efectividad de las vacunas para cualquier forma severa de COVID-19 después de los 6 meses de la vacunación. Si bien se ha notificado cierta reducción de la eficacia de las vacunas 
para algunas VOC (como la B.1.617.2), estudios recientes sugieren que el riesgo de infección después del esquema de vacunación completo se da principalmente en individuos inmunodeprimidos. Además, refieren que, si bien las infecciones en personas vacunadas son aún esperables, la mayoría son menos graves que las observadas en personas no vacunadas.

Tercero, el suministro global de vacunas y equidad global y nacional. La OMS sostiene que, el priorizar las dosis de refuerzo por encima de una mayor rapidez y amplitud en la cobertura de la vacunación inicial (primaria), puede representar un riesgo importante para la mitigación global de la pandemia. Además, refiere que las decisiones políticas para agregar una dosis de refuerzo en los programas nacionales de vacunación deben ser tomadas en base a una evidencia sólida y considerando la disponibilidad mundial de vacunas.

Con sustento en la evaluación de los tres factores mencionados anteriormente, la OMS considera que la evidencia actual sigue siendo limitada y no concluyente para recomendar la administración de dosis de refuerzo a la población en general.

Belgian Health Care Knowledge Centre. "Rapid review of the evidence on a COVID-19 booster dose after a primary vaccination schedule" (26)

Se trata de una revisión rápida de la evidencia sobre dosis de refuerzo para COVID-19 realizada por el Grupo de Trabajo para Vacunación, constituido por diferentes instituciones de salud y centros de investigación de Bélgica. La fecha del reporte fue el 17 de agosto, 2021.

La búsqueda de evidencia para este documento se realizó en la base de datos PubMed y en el repositorio de preimpresiones medRxiv, aunque también se incluyeron algunos artículos identificados por parte de los expertos. Se presentan las diferentes estrategias de búsqueda y proceso de selección de estudios para cada uno de los siguientes tópicos: infecciones después de la vacunación ("breakthrough infections"), efectividad y respuesta inmune después de dos dosis de vacuna, seguridad de esquemas de vacunación heteróloga para COVID-19, y efectividad y seguridad después de tres dosis de vacuna para COVID-19.

Los autores incluyeron un total de siete estudios como parte de la evidencia para respuesta inmune y seguridad de la dosis de refuerzo. El motivo de inclusión de resultados de inmunidad y no de efectividad, fue porque los autores no encontraron estudios de esta última al momento de la búsqueda. De los estudios considerados, seis de ellos incluyeron a participantes que habían recibido su esquema de vacunación primaria con la vacuna BNT162b2 y tenían algún grado de inmunosupresión, lo que implica que la intervención fue una dosis adicional y no una de refuerzo (incluso uno de ellos consideró como tercera dosis la vacuna mRNA-1273 de Moderna). El último estudio, que se realizó en adultos sanos y evaluó una dosis de refuerzo, no se realizó en la población considerada en el presente reporte pues la vacuna evaluada fue CoronaVac (tanto en el esquema primario como para la dosis de refuerzo), la cual no es parte del esquema de inmunización para COVID-19 en Perú.
El Grupo de Trabajo, de acuerdo con la evidencia revisada, consideró como aceptable la administración selectiva de una dosis adicional de tipo ARN-m en personas inmunodeprimidas, con inmunodeficiencias congénitas, en hemodiálisis crónica, con $\mathrm{VIH}$, con hemopatías malignas u otros cánceres (que hayan estado en tratamiento durante el último año), que hayan recibido un trasplante de órganos o células madre y en aquellos con enfermedades inflamatorias y tratados con inmunosupresores. Para las personas de edad avanzada consideran que, si bien la respuesta inmune puede ser más débil, aún no hay estudios publicados sobre la administración de una dosis adicional o de refuerzo. Sin embargo, mencionan que las personas de edad avanzada que además vivan en centros de vida asistida o asilos y se hayan vacunado antes que sus contrapartes que viven en la comunidad podrían recibir una dosis adicional $(<6$ meses después de la vacunación primaria) o una dosis de refuerzo ( $>6$ meses). El Grupo de Trabajo considera que se debe esperar evidencia adicional para considerar una extensión gradual de la dosis de refuerzo a otros grupos de riesgo.

Comité Asesor en Vacunas y Estrategias de Inmunización (CAVEI). "Recomendación del CAVEI sobre la introducción de una dosis de refuerzo al plan de vacunación COVID19 ")(27)

Se trata de las recomendaciones por parte del CAVEI sobre el uso de una dosis de refuerzo de vacuna COVID-19 en población chilena. La fecha fue el 29 de julio de 2021.

Realizaron una revisión de la información disponible a la fecha, respecto a la situación epidemiológica de COVID-19, la protección otorgada y la seguridad de la vacunación COVID19 en Chile, la evidencia sobre dosis de refuerzo de esquemas de vacunación COVID-19 y la situación de abastecimiento de vacunas COVID-19. Sin embargo, no se describe en el documento cómo fue que se hizo el proceso de selección de evidencia.

Los documentos incluidos como parte de la evidencia de una dosis de refuerzo para COVID-19 incluyeron un estudio que evaluó la tercera dosis de BNT162b2 en receptores de trasplante de órganos con esquema de inmunización primaria con la misma vacuna, una preimpresión de un ensayo clínico fase II sobre inmunogenicidad y seguridad de una tercera dosis de CoronaVac después de una vacunación primaria con la misma vacuna, una preimpresión sobre tolerabilidad e inmunogenicidad de una tercera dosis de ChAdOx1 nCoV-19 después de una vacunación primaria con la misma vacuna, una nota de prensa sobre dosis de refuerzo de CanSino, un comunicado de Pfizer-BioNTech del 8 de julio 2021 y un comunicado conjunto de los CDC y la FDA sobre la necesidad de dosis de refuerzo en Estados Unidos. Consideraron también información sobre algunos estudios en curso, como el COV-Boost de Reino Unido y un ensayo clínico fase I/II del Instituto Nacional de Alergias y Enfermedades Infecciosas (NIAID, por sus siglas en inglés).

Los miembros del CAVEI, de acuerdo con la evidencia revisada, recomendaron introducir una dosis de refuerzo con una vacuna de tipo ARN-m en la campaña de vacunación contra COVID-19 en Chile. Los autores resaltan que, si bien la evidencia sobre dosis de refuerzo es escasa, uno de los aspectos que motiva esta recomendación es la aparente 
Revista del Cuerpo Médico del HNAAA, Vol. 14 Supl. № 1 ( 2021) | SARS-CoV-2, COVID-19 y Pandemia

tendencia a la disminución de la efectividad de la vacuna CoronaVac en el país. Es importante precisar que, si bien el programa de inmunización contra COVID-19 en Chile incluye también las vacunas de Pfizer y AstraZeneca, al 15 de julio, más del 70\% de la población estaba vacunada con CoronaVac.

Respecto a la priorización, recomiendan que la aplicación de dosis de refuerzo inicie con aquellos con mayor riesgo de infección severa o muerte, y con mayor tiempo desde el esquema completo de vacunación, incluyendo a los pacientes con algún grado de inmunosupresión, trabajadores de salud, personas de edad avanzada en residencias de larga estadía, personas en privación de libertad, personas con comorbilidades e individuos que cumplen labores importantes relacionadas al funcionamiento del país. Adicionalmente, refieren que el refuerzo debe estar principalmente dirigido a personas cuyo esquema primario haya sido con una vacuna distinta al tipo ARN-m.

\section{ii). Estudios observacionales}

Bar-On YM et al, 2021. "BNT162b2 vaccine booster dose protection: A nationwide study from Israel" (28)

El diseño del estudio fue de cohorte dinámica y su objetivo fue evaluar la efectividad de una dosis de refuerzo con la vacuna BNT162b2 para la prevención de infección confirmada y casos severos de COVID-19. La población de estudio estuvo conformada por adultos de 60 años a más con esquema completo de inmunización para COVID-19 (i.e. haber recibido las dos dosis de la vacuna) hace al menos cinco meses (antes del 01 de marzo de 2021). No se consideró a las personas que carecían de información sobre su sexo, que estaban en el extranjero en agosto de 2021, que habían sido infectados con COVID-19 antes del 30 de julio de 2021, que recibieron una dosis de refuerzo antes del 30 de julio de 2021, o se vacunaron completamente antes del 16 de enero.

Todos los datos fueron extraídos de la base de datos del Ministerio de Salud de Israel, incluyendo las fechas de vacunación (primera, segunda y tercera dosis), las pruebas RT-PCR (fechas y resultados), la fecha de hospitalización por COVID-19, el estado clínico (leve, severo), y variables sociodemográficas como la edad, sexo y grupo demográfico (judío general, árabe, judío ultraortodoxo). La infección confirmada se definió como un resultado de RT-PCR positivo, y la enfermedad severa, como una frecuencia respiratoria en reposo $>30$ respiraciones/ $\mathrm{min}$, o SpO2 en aire ambiente $<94 \%$, o relación entre $\mathrm{PaO} 2$ y $\mathrm{FiO} 2<300$.

El período de estudio inició el 30 de julio de 2021 (día en el que se inició la campaña de vacunación con dosis de refuerzo en Israel) y culminó el 22 de agosto. Los resultados de interés se evaluaron en dos cohortes. La primera cohorte estuvo conformada por personas que recibieron dos dosis de vacuna y la dosis de refuerzo ("cohorte de refuerzo"). La segunda estuvo conformada por personas que solo recibieron dos dosis de vacuna ("cohorte sin refuerzo"). Dado que las cohortes eran dinámicas, los individuos que pertenecían inicialmente a la segunda cohorte, la abandonaban al recibir la dosis de refuerzo e ingresaban a la primera cohorte 12 días después; siempre que no tuvieran una infección confirmada en el periodo intermedio. Para cada persona de la "cohorte con refuerzo", los días de riesgo comenzaron al entrar en la cohorte (12 días después de recibir la tercera dosis), y terminaron con la aparición de alguno de los desenlaces de interés o al final del periodo de estudio. En el caso de la "cohorte sin refuerzo", los días de riesgo empezaron al principio del periodo de estudio y terminaron con la aparición de algún desenlace de interés o al final del periodo de estudio.

Para evaluar el cálculo de las razones de incidencia de los desenlaces de interés, los autores construyeron modelos de regresión de Poisson, ajustando por edad, sexo, grupo demográfico y fecha de la dosis de la segunda vacuna. Para estimar el efecto de la dosis de refuerzo, los autores calcularon la reducción del riesgo relativo (RRR) dividiendo la unidad entre el exponente del coeficiente de regresión asociado a la cohorte de tratamiento. Adicionalmente, los autores realizaron diferentes análisis de sensibilidad usando técnicas de emparejamiento por diferentes variables para disminuir la confusión, y modelando los datos en función del tiempo desde la dosis de refuerzo.

\section{Resultados del estudio}

Se incluyeron en el análisis un total de 1,144,690 adultos de 60 años a más. En la "cohorte sin refuerzo" hubo 4,018,929 personas-días en riesgo con 3,473 infecciones confirmadas y 330 casos severos de COVID-19, y 3,351,598 personas-días en la "cohorte con refuerzo" con 313 infecciones confirmadas y 32 casos severos. La "cohorte con refuerzo" tuvo una mayor proporción de varones ( $50 \%$ vs $43 \%$ ), judíos en general (93\% vs $82 \%$ ), personas mayores (60\% vs $47 \%$ de 70 años a más) y personas que fueron vacunadas antes ( $79 \%$ vs $40 \%$ vacunados en la última quincena de enero).

\section{Infección confirmada}

El principal resultado fue un RRR de 11,4 (IC 95\%: 10 - 12,9) para infección confirmada (a favor de la dosis de refuerzo). El primer análisis de sensibilidad, que ajustó por características individuales siguiendo la misma metodología de Dagan et $\mathrm{al}^{(29)}$, dio como resultado un RRR de 13,4 (IC 95\%: 8,2 - 21,4). El segundo análisis de sensibilidad, que emparejó por días, reportó un RRR de 9,6 (IC 95\%: 8,1 - 11,4). Un análisis adicional, que comparó el riesgo de infección durante los 12 a más días después de la dosis de refuerzo y 4-6 días después de la dosis de refuerzo, encontró un RRR de 4,7 (IC 95\%: 4 - 5,4).

Los autores también presentaron una gráfica con los resultados de la regresión de Poisson inicial, considerando el número de días después de la dosis de refuerzo como covariable adicional. Según la gráfica, existió una aparente protección inicial que fue disminuyendo en el transcurso de la primera semana hasta que, a partir del séptimo día, comenzó a incrementar. Sin embargo, solo se grafican los datos obtenidos hasta el día 16 después de la vacunación de refuerzo debido a los amplios intervalos de confianza.

\section{Severidad}

Para las personas con 12 a más días entre la vacunación de refuerzo y la enfermedad severa, se reportó un RRR de 15,5 (IC 95\%: 10,5 - 22,8). Un análisis de sensibilidad subsiguiente, que emparejó por días, evidenció un RRR de 9.5 (IC 95\%: 5 19.6) también a favor de la dosis de refuerzo.

\section{Seguridad}

El estudio no evaluó eventos adversos. 
Revista del Cuerpo Médico del HNAAA, Vol. 14 Supl. №1 ( 2021 ) | SARS-CoV-2, COVID-19 y Pandemia

\section{Análisis}

Las diferencias existentes entre las características basales de los sujetos de cada cohorte sugieren la presencia de confusión residual. Si bien los autores controlaron por algunas variables en sus modelos de regresión e hicieron análisis de sensibilidad que incluyeron emparejamiento por estas variables, esto no garantiza la eliminación del efecto confusor. Primero, porque algunas de las variables, como grupo demográfico y momento en el cual se vacunaron, no solo han demostrado ser confusores fuertes en la misma población de estudio, sino también estar relacionadas entre $\mathrm{si}^{\prime(30)}$. Segundo, el emparejamiento por variables para disminuir la confusión puede resultar útil cuando se cuenta con un listado considerable de variables que puedan funcionar como potenciales confusoras; sin embargo, cuando la cantidad de variables es limitada como lo fue en el estudio (independientemente de la causa), los resultados finales del análisis, con o sin emparejamiento, pueden incluso no diferir sustancialmente ${ }^{(31)}$. Tercero, los autores tampoco presentan información sobre cómo se hizo la evaluación del balance de covariables ni cuál fue el resultado después del emparejamiento. Por otra parte, la presencia de sesgo oculto por confusión no medida, que no puede ser controlado por ninguna de las técnicas anteriormente mencionadas, es también muy probable. Algunas de estas variables no incluidas en el análisis incluyen la búsqueda de atención médica, conductas de prevención, comorbilidades, entre otros.

Otro sesgo probable es el de comportamiento ("behavioral bias"), el cual puede incluir una menor realización de pruebas diagnósticas (RT-PCR) y mayores conductas de prevención en las personas que recibieron la dosis de refuerzo, al menos en los primeros días posteriores a la dosis. A esto se le suma las diferencias mencionadas en el párrafo anterior y el hecho de que, según hallazgos de los autores, en el primer día ya había una reducción de 5-7 veces el riesgo de tener infección. Todos estos escenarios incrementan considerablemente la posibilidad de sesgo de selección.

Los autores realizan un análisis secundario en el cual comparan la tasa de infección en los días 4-6 y 12 a más, únicamente en la cohorte que recibió la dosis de refuerzo. En este análisis, que además permite controlar mejor el sesgo de selección y tendría mayor aplicabilidad, se evidenció que la reducción del riesgo relativo fue de 4,7 veces (IC 95\%: 4 $5,4)$, mucho menor al resultado inicialmente reportado de 11,4 veces (IC 95\%: 10 - 12,9). Este escenario motiva el planteamiento de las siguientes observaciones. Primero, si este análisis de sensibilidad controla mejor el sesgo de selección (que es una limitación importante de los análisis primarios), llama la atención por qué no se realiza este mismo análisis para el desenlace de severidad, que es un desenlace de mayor relevancia. Segundo, los autores no detallan por qué escogen los días 4-6 y no, por ejemplo, los días 1-6. Esto es importante porque los días 4-6 resultan ser los de menor reducción relativa de riesgo, lo que estaría inflando el efecto de la dosis de refuerzo cuando se compara con los días 12 a más.

El estudio también está sujeto a sesgo de persona-tiempo inmortal ("inmortal person-time bias"), más aún por el diseño que usaron (cohorte dinámica). Esto es particularmente importante porque implica que algunos sujetos contribuyan con días en riesgo ("days at risk") durante los cuales les habría sido imposible experimentar el evento de interés ${ }^{(32,33)}$. Independientemente de que los autores mencionan que las personas en los días 0 a 12 después de la dosis de refuerzo no contribuyeron en los días en riesgo, el sesgo puede estar igual presente puesto que las personas no solo son "inmortales" en el período señalado. También son "inmortales" antes de la dosis de refuerzo si se trata de una persona que eventualmente recibió esta dosis, porque si se hubiera infectado, hospitalizado o muerto, no habría recibido el refuerzo. Entonces, en términos de análisis de datos, cuando este tiempo de persona-tiempo inmortal es incluido en el denominador para el cálculo de razón de incidencias del desenlace de interés, el resultado terminaría favoreciendo sustancialmente al grupo que recibió la dosis de refuerzo.

Finalmente, la definición usada para severidad difiere de la considerada en el presente reporte breve. La diferencia subyace, principalmente, en que el criterio usado por los autores no incluye la presencia de signos clínicos de neumonía como criterio necesario (como es sugerido por la OMS $)^{(34)}$. Esto no solo limita la validez externa de los resultados, sino también podría estar sobreestimando el verdadero efecto de la dosis de refuerzo para un verdadero estado de severidad. Cabe resaltar que una adecuada definición de severidad es importante para poder tener una mejor proyección sobre otros desenlaces de interés como hospitalización ${ }^{(17)}$, ingreso a $\mathrm{UCl}(35)$ y riesgo de muerte ${ }^{(36)}$.

\section{iii) Ensayos clínicos fase III en curso o aún no publicados}

Study to Describe the Safety, Tolerability, Immunogenicity, and Efficacy of RNA Vaccine Candidates Against COVID-19 in Healthy Individuals (NCT04368728)

Es un estudio de fase $1 / 2 / 3$, aleatorizado, controlado con placebo, triple ciego, con sedes en Argentina, Brazil, Alemania, Sudáfrica, Turquía y Estados Unidos, con una proyección estimada de 43,998 participantes sanos a enrolar. Específicamente para la fase 3 , se evaluará la eficacia y seguridad de la dosis de refuerzo en población de 18 a 55 años. Para ello, un subconjunto de participantes de esta fase recibirá una tercera dosis de $30 \mu \mathrm{g}$ de BNT162b2 o $30 \mu \mathrm{g}$ de BNT162b2SA, y otro subconjunto de participantes recibirá una tercera dosis más baja (5 o $10 \mu \mathrm{g})$ de BNT162b2. Los desenlaces primarios incluyen el reporte de eventos adversos (serios y no serios) y la no inferioridad de los títulos de anticuerpos neutralizantes después de una tercera dosis de BNT162b2 a $30 \mu \mathrm{g}$ en comparación con después de 2 dosis de BNT162b2, en los mismos individuos. Fecha aproximada de término del estudio: mayo del 2023.

Study to Evaluate the Safety and Efficacy of a Booster Dose of BNT162b2 Against COVID-19 in Participants $\geq 16$ Years of Age (NCT04955626)

ECA fase III triple ciego actualmente en curso. Tiene proyectado el enrolamiento de 10,000 participantes sanos de 16 años a más de Estados Unidos, Brasil y Sudáfrica. El estudio evaluará la seguridad, tolerabilidad y eficacia de una dosis de refuerzo $(30 \mu \mathrm{g})$ de BNT162b2, administrada a participantes que hayan recibido previamente 2 dosis de BNT162b2 al menos 6 meses antes de la aleatorización. La duración del 
Revista del Cuerpo Médico del HNAAA, Vol. 14 Supl. № 1 ( 2021) | SARS-CoV-2, COVID-19 y Pandemia

estudio para cada participante será de aproximadamente 12 meses. Los desenlaces primarios incluyen la incidencia de casos confirmados de COVID-19 (con y sin infección previa por SARS-CoV-2) y los eventos adversos (serios y no serios). Fecha aproximada de término del estudio: septiembre del 2022.

\begin{abstract}
A Phase 3 Study to Evaluate the Safety, Tolerability, and Immunogenicity of Multiple Production Lots and Dose Levels of BNT162b2 RNA-Based COVID-19 Vaccines Against COVID-19 in Healthy Participants (NCT04713553)

ECA fase III triple ciego, registrado como completado y realizado en 1,530 participantes sanos de 18 a 50 años. Se evaluará la seguridad, tolerabilidad e inmunogenicidad de la dosis de refuerzo con dos vacunas ARN-m (BNT162b2 y BNT162b2.B.1.351) en dosis de $30 \mu \mathrm{g}$. Los desenlaces primarios incluyen la media geométrica de los títulos de anticuerpos neutralizantes y los eventos adversos (serios y no serios). Según la página de clinicaltrials.gov, el estudio concluyó el 22 de julio del 2021, pero aún no hay resultados publicados
\end{abstract}

\section{Discusión}

\section{Hallazgos principales}

El presente estudio buscó evaluar la mejor evidencia disponible, al 13 de septiembre, sobre la eficacia y seguridad de la dosis de refuerzo con la vacuna BNT162b2, en población que haya recibido el esquema de inmunización completa (dos dosis) con la vacuna BBIBP-CorV, BNT162b2 o ChAdOx1 nCoV19. A través de una búsqueda sistemática de la literatura, se identificaron cuatro documentos de recomendación $(20,24,26,27)$, un estudio observacional realizado en Israel (28) y tres ensayos clínicos en curso (NCT04368728, NCT04955626 y NCT04713553).

\section{Sobre los documentos de recomendación}

Los documentos revisados coinciden en que existe aún limitada evidencia respecto al uso de una dosis de refuerzo. Esto va en línea con el hecho de que la mayoría de los estudios actualmente disponibles son en población con algún grado de inmunosupresión ${ }^{(37-43)}$, lo cual hace que la recomendación a favor sea para una dosis adicional en este grupo específico, y no así para una dosis de refuerzo en la población en general. Si bien el Comité Asesor en Vacunas y Estrategias de Inmunización de Chile recomienda actualmente el uso de una dosis de refuerzo contra COVID-19, uno de sus motivos principales es la aparente disminución de la efectividad de la vacuna CoronaVac, la cual ha sido aplicada a más del $70 \%$ de la población del país. Sin embargo, es importante resaltar que la evidencia aún es limitada respecto a la disminución de la inmunidad en personas con esquema de inmunización completa para COVID-19. Además, existen reportes que sugieren que la vacunación primaria, pese a que pueda tener una reducción en su efectividad a lo largo del tiempo, mantiene niveles adecuados para evitar la infección por SARS-CoV-2 y también para casos severos, que es un desenlace de mayor relevancia clínica ${ }^{(44)}$. Este escenario se mantiene incluso en la presencia de variantes de mayor transmisibilidad como la B.1.617.2 $2^{(45,46)}$.

\section{Sobre el estudio observacional}

El estudio realizado en Israel ${ }^{(28)}$ tiene diferentes limitaciones metodológicas que ya han sido mencionadas en el presente manuscrito. A esto se le suma que el estudio tuvo un seguimiento promedio limitado (7 días-persona) y, aún más importante, el efecto a corto plazo evidenciado no garantiza un beneficio a largo plazo. Por otra parte, el estudio tampoco evaluó eventos adversos. Esto es particularmente importante ya que, si bien las vacunas actualmente disponibles han demostrado ser seguras a corto y mediano plazo, no existen estudios clínicos publicados que hayan evaluado la seguridad de una dosis de refuerzo. Dado que algunas vacunas han demostrado tener fuertes respuestas inmunitarias, en el caso de requerirse eventualmente una vacunación de refuerzo, esta podría generar una adecuada respuesta inmunológica con dosis menores a la de la vacunación primaria ${ }^{(47)}$. Además, es importante mencionar que una respuesta inmune más fuerte puede relacionarse con reacciones adversas inflamatorias después de la vacunación ${ }^{48}$. Por este motivo, son necesarios ensayos clínicos -varios de ellos se encuentran actualmente en curso- que evalúen de manera adecuada y con un estricto sistema de vigilancia la seguridad de dosis adicionales o de refuerzo.

\section{Otras consideraciones}

Los estudios que evalúen la eficacia o efectividad de una dosis de refuerzo deberían priorizar en sus objetivos la severidad de la enfermedad ${ }^{(20,47)}$. De esta manera se puede hacer una mejor proyección sobre otros desenlaces como hospitalización, necesidad de $\mathrm{UCl}$ y muerte, y así poder tomar una mejor decisión en base a una necesidad real, con implicancias importantes en la salud pública y el uso de recursos humanos y económicos. Sin embargo, como se ha mencionado previamente, la evidencia actualmente disponible no parece sustentar la necesidad de una dosis de refuerzo -al menos por ahora-, puesto que la eficacia de la vacunación primaria sigue siendo alta para la prevención de casos severos.

\section{Conclusión}

En un escenario donde aún existen recursos limitados, es necesario contar con evidencia sólida y de buena calidad para poder tomar decisiones de salud pública que además sean tiempo-dependientes (por ejemplo, en etapas iniciales de una eventual futura "ola"). Al 13 de septiembre de 2021, no existe evidencia suficiente sobre la eficacia de agregar una dosis de refuerzo al esquema de inmunización para COVID-19. La evidencia disponible no permite justificar el uso de una dosis de refuerzo con la vacuna BNT162B2 en población que haya recibido previamente dos dosis de la vacuna BBIBP-CorV, BNT162b2 o ChAdOx1 nCoV-19. Por tal motivo, su administración podría considerarse, pero en el contexto de un ensayo clínico.

En el contexto peruano, el objetivo principal de la estrategia de vacunación debería ser maximizar la cobertura de la población vacunada con dos dosis, pues aún no se ha alcanzado el $50 \%$ de inmunización completa en la población total de ninguna región. La eventual inclusión de una dosis de refuerzo como medida de salud pública debería estar justificada en evidencia sólida de su eficacia en la reducción de muertes y/o enfermedad severa (ej. necesidad de UCI o ventilación mecánica) que permita tomar una decisión no solo respecto a la pertinencia de su uso, sino también al público objetivo y al momento adecuado para su aplicación.

\section{REFERENCIAS BIBLIOGRÁFICAS}

1. World Health Organization. WHO Coronavirus (COVID-19) Dashboard 
[acceso: 13 de septiembre, 2021]. Geneva: WHO; 2021.

2. Presidencia del Consejo de Ministros. Plataforma Nacional de Datos Abiertos. Lima, Perú: PCM; 2021.

3. Polack FP, Thomas SJ, Kitchin N, Absalon J, Gurtman A, Lockhart S, et al. Safety and Efficacy of the BNT162b2 mRNA Covid-19 Vaccine. N Engl J Med. 2020;383(27):2603-15.

4. Baden LR, El Sahly HM, Essink B, Kotloff K, Frey S, Novak R, et al. Efficacy and Safety of the mRNA-1273 SARS-CoV-2 Vaccine. N Engl J Med. 2021;384(5):403-16.

5. Akova M, Unal S. A randomized, double-blind, placebo-controlled phase III clinical trial to evaluate the efficacy and safety of SARS-CoV-2 vaccine (inactivated, Vero cell): a structured summary of a study protocol for a randomised controlled trial. Trials. 2021;22(1):276.

6. Al Kaabi N, Zhang Y, Xia S, Yang Y, Al Qahtani MM, Abdulrazzaq N, et al. Effect of 2 Inactivated SARS-CoV-2 Vaccines on Symptomatic COVID-19 Infection in Adults: A Randomized Clinical Trial. Jama. 2021;326(1):3545.

7. Shinde V, Bhikha S, Hoosain Z, Archary M, Bhorat Q, Fairlie L, et al. Efficacy of NVX-CoV2373 Covid-19 Vaccine against the B.1.351 Variant. N Engl J Med. 2021;384(20):1899-909.

8. Knoll MD, Wonodi C. Oxford-AstraZeneca COVID-19 vaccine efficacy. Lancet. 2021;397(10269):72-4.

9. Shay DK, Gee J, Su JR, Myers TR, Marquez P, Liu R, et al. Safety Monitoring of the Janssen (Johnson \& Johnson) COVID-19 Vaccine United States, March-April 2021. MMWR Morb Mortal Wkly Rep. 2021;70(18):680-4.

10. Euronews \& AP. Europe starts COVID booster shots as WHO says jabs 'should be saved for developing world [Internet]. [Acceso: 23 de s e p t i e m b r e, $\quad 20221$ l. D is p o n ible e n : https: / /www.euronews.com/2021/09/01/booster-shots-of-covid-19vaccine-should-be-saved-for-developing-world-who-chief.

11. Channel news Asia. Singapore to expand COVID-19 vaccine booster programme to cover those aged between 50 and 59 [Internet]. [Acceso: 25 de septiembre, 2021 ]. Disponible en: https://www.channelnewsasia.com/singapore/singapore-expandcovid-19-vaccine-booster-programme-aged-between-50-and-592199531.

12. US Food \& Drug Administration. FDA News Release: Coronavirus (COVID19) Update: FDA Authorizes Additional Vaccine Dose for Certain Immunocompromised Individuals. Disponible en: h t t p s: / / w w w. f d a. gov/ n e w s - event s / press announcements/coronavirus-covid-19-update-fda-authorizes additional-vaccine-dose-certain-immunocompromised.

13. Reuters. Dominican Republic giving third COVID-19 vaccine dose in move to stave off new virus variants [Internet]. [Acceso: 23 de septiembre, 2021]: Disponible en: https://www.reuters.com/business/healthcarepharmaceuticals/dominican-republic-giving-third-covid-19-vaccinedose-move-stave-off-new-virus-2021-07-01/.

14. Reuters. Chile to give COVID-19 vaccine boosters for those inoculated with Sinovac [Internet]. [Acceso: 25 de septiembre, 2021]. Disponible en: https://www.reuters.com/world/americas/chile-give-covid-19vaccine-boosters-those-inoculated-with-sinovac-2021-08-05/.

15. The Brazilian report. Uruguay's vaccine booster campaign advancing fast [Internet]. [Acceso: 25 de septiembre, 2021]. Disponible en: https://brazilian.report/liveblog/2021/09/01/uruguay-booster-fast/.

16. Centers for Disease Control and Prevention. Interim Clinical Considerations for Use of COVID-19 Vaccines Currently Approved or Authorized in the United States. USA: CDC; 2021.

17. Centers for Disease Control and Prevention. Interim Clinical Guidance for Management of Patients with Confirmed Coronavirus Disease (COVID19). USA: CDC; 2021.

18. Instituto de Investigación en Tecnologías en Salud e Ivestigación. Reporte Breve $\mathrm{N}^{\circ}$ 04-2021: Evaluación de la eficacia y seguridad de la vacunación de refuerzo con la vacuna BNT162b2 (comirnaty), en pacientes que recibieron el esquema de vacunación completa con la vacuna BBIBP-CorV (sinopharm). Lima, Perú: IETSI-EsSalud; 2021.

19. Presidencia del Consejo de Ministros. Coronavirus: vacunas contra la COVID-19 en el Perú. Lima, Perú: PCM; 2021.

20. European Centre for Disease Prevention and Control. Interim public health considerations for the provision of additional COVID-19 vaccine doses, 1 September 2021. Estocolmo: ECDC; 2021.

21. International Vaccine Access Center JHBSoPH, World Health Organization. Results of COVID-19 Vaccine Effectiveness Studies: An Ongoing Systematic Review, Updated August 12, 2021.

22. MCMaster University Health Forum C-ENDEN. COVID-19 Living Evidence Synthesis \#6 (Version 15: 11 August 2021). 2021.

23. European Centre for Disease Prevention and Control. Partial COVID-19 vaccination, vaccination following SARS-CoV-2 infection and heterologous vaccination schedule: summary of evidence, 22 July 2021 2021.

24. World Health Organization. Interim statement on COVID-19 vaccine booster doses, 10 August 2021. Geneva: WHO; 2021.

25. Plotkin SA. Vaccines: correlates of vaccine-induced immunity. Clinical Infectious Diseases: An Official Publication of the Infectious Diseases Society of America. 2008;47(3):401-9.

26. Belgian Health Care Knowledge Center. Rapid review of the evidence on a COVID-19 booster dose after a primary vaccination schedule, 17 de agosto, 2021. Belgium: KCE; 2021.

27. Comité Asesor en Vacunas y Estrategias de Inmunización. Recomendación del CAVEI sobre la introducción de una dosis de refuerzo al plan de vacunación COVID-19, 29 de julio 2021. 2021.

28. Bar-On YM, Goldberg Y, Mandel M, Bodenheimer O, Freedman L, Kalkstein $\mathrm{N}$, et al. BNT162b2 vaccine booster dose protection: A nationwide study from Israel. medRxiv. 2021.

29. Dagan N, Barda N, Kepten E, Miron O, Perchik S, Katz MA, et al. BNT162b2 mRNA Covid-19 Vaccine in a Nationwide Mass Vaccination Setting. N Engl J Med. 2021

30. Goldberg Y, Mandel M, Bar-On YM, Bodenheimer O, Freedman L, Haas EJ, et al. Waning immunity of the BNT162b2 vaccine: A nationwide study from Israel. medRxiv. 2021.

31. Faresjö T, Faresjö À. To Match or Not to Match in Epidemiological Studies-Same Outcome but Less Power. Int J Environ Res Public Health. 2010;7(1):325-32.

32. Suissa S. Immortal Time Bias in Pharmacoepidemiology. American Journal of Epidemiology. 2008;167(4):492-9.

33. Schmidt SaJ, Lash TL. Immortal person-time bias in the association between herpes zoster and survival following autologous stem cell transplantation. Bone Marrow Transplant. 2015;50(6):878-9.

34. World Health Organization. COVID-19 clinical management: living guidance, 25 January 2021. Geneva: WHO; 2021.

35. Llitjos J-F, Bredin S, Lascarrou J-B, Soumagne T, Cojocaru M, Leclerc M, et al. Increased susceptibility to intensive care unit-acquired pneumonia in severe COVID-19 patients: a multicentre retrospective cohort study. Annals of Intensive Care. 2021;11(1):20.

36. Zhou F, Yu T, Du R, Fan G, Liu Y, Liu Z, et al. Clinical course and risk factors for mortality of adult inpatients with COVID-19 in Wuhan, China: a retrospective cohort study. The Lancet. 2020;395(10229):1054-62.

37. Albach FN, Burmester GR, Biesen R. Successful BNT162b2 booster vaccinations in a patient with rheumatoid arthritis and initially negative antibody response. Annals of the Rheumatic Diseases. 2021;80(10):1361-2.

38. Chavarot N, Morel A, Leruez-Ville M, Vilain E, Divard G, Burger C, et al. Weak antibody response to three doses of mRNA vaccine in kidney transplant recipients treated with belatacept. Am J Transplant. 2021.

39. Re D, Seitz-Polski B, Carles M, Brglez V, Graça D, Benzaken S, et al. Humoral and cellular responses after a third dose of BNT162b2 vaccine in patients with lymphoid malignancies. medRxiv. 2021

40. Del Bello A, Abravanel F, Marion O, Couat C, Esposito L, Lavayssière L, et al. Efficiency of a boost with a third dose of anti-SARS-CoV-2 messenger RNA-based vaccines in solid organ transplant recipients. Am J Transplant. 2021

41. Kamar N, Abravanel F, Marion O, Couat C, Izopet J, Del Bello A. Three Doses of an mRNA Covid-19 Vaccine in Solid-Organ Transplant Recipients. N Engl J Med. 2021;385(7):661-2.

42. Longlune N, Nogier MB, Miedougé M, Gabilan C, Cartou C, Seigneuric B, et al. High immunogenicity of a messenger RNA based vaccine against SARS-CoV-2 in chronic dialysis patients. Nephrol Dial Transplant. 2021.

43. Stumpf J, Tonnus W, Paliege A, Rettig R, Steglich A, Gembardt F, et al. Cellular And Humoral Immune Responses after Three Doses of BNT162b2 mRNA SARS-Cov-2 Vaccine in Kidney Transplant. Transplantation. 2021.

44. Thomas SJ, Moreira ED, Kitchin N, Absalon J, Gurtman A, Lockhart S, et al. Safety and Efficacy of the BNT162b2 mRNA Covid-19 Vaccine through 6 Months. New England Journal of Medicine. 2021.

45. Pouwels KB, Pritchard E, Matthews PC, Stoesser N, Eyre DW, Vihta K-D, et al. Impact of Delta on viral burden and vaccine effectiveness against new SARS-CoV-2 infections in the UK. medRxiv. 2021.

46. Fowlkes A. Effectiveness of COVID-19 Vaccines in Preventing SARS-CoV-2 Infection Among Frontline Workers Before and During B.1.617.2 (Delta) Variant Predominance - Eight U.S. Locations, December 2020-August 2021. MMWR Morb Mortal Wkly Rep. 2021;70.

47. Krause PR, Fleming TR, Peto R, Longini IM, Figueroa JP, Sterne JAC, et al. Considerations in boosting COVID-19 vaccine immune responses. The Lancet. 2021.

48. Zhuang C-L, Lin Z-J, Bi Z-F, Qiu L-X, Hu F-F, Liu X-H, et al. Inflammationrelated adverse reactions following vaccination potentially indicate a stronger immune response. Emerg Microbes Infect. 2021;10(1):365-75. 